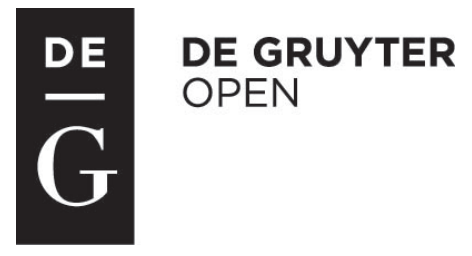

Przedsiębiorczość i Zarządzanie Entrepreneurship and Management University of Social Sciences Publishing House

ISSN 1733-2486

Volume XV, Issue 2, pp. 51-61

DOI 10.2478/eam-2014-0016

Zofia Warzyńska-Bartczak

Medical University of Lodz

\title{
Psychiatrists and patients suffering from depressive disorders and schizophrenia in one organization basing on the hospitals in Lodz region
}

\begin{abstract}
Collaboration between doctors and patient is a perfect example of a specific kind of collaboration in the organization. Difficulties with establish proper relations between psychiatrists and patients with depressive disorders and schizophrenia is the subject of the paper. The thesis of the work assumes that psychiatrists and patients belong to the totally different "worlds" what is the cause of inability to create proper mutual collaboration between them. In 2009 a questionnaire survey was conducted at public mental hospitals of the Lodz region. Both doctors and patients notice a number of communication barriers and factors hampering the mutual cooperation, at the same time demonstrating the will to agree and cooperate. The proper contact and collaboration affect mental disposition and motivation for the process of recovery from the illness.

Conclusion

To a large extent not only a course of the treatment, but also a correct social functioning of both groups of the patients depends on the quality of the interrelation between them and psychiatrists. It appears, however, that the hierarchy of obstacles of successful therapy is completely different among the doctors and patients.
\end{abstract}

Key words: doctor-patient collaboration, depression, schizophrenia, "two worlds".

\section{Background}

Many authors emphasize how important collaborative organization and partnership are [Hill, Morgan 2012], also in the medical organizations [World Health Organization, 2004]. The specific character of a doctor-patient relation- 
ship is underlined by many authors, physicians, psychologists and therapists, see e.g. [Beach et al. 2006, pp. 347-354; Pulia 2011, pp. 18-19; Roter 1977, pp. 281-315; Roter et al 1987, pp. 437-451; Roter 2006, pp. S28-34; Salmon 2000; Salmon 2006, pp. 137-159; Sofaer, Firminger 2005, pp. 513-559]. One should notice that not always the situation was as clear as it seems to be now when both patients and health professionals are aware of the fundamental conditions of the successful process of recovery. Nowadays, specialists and patients know how profoundly the beliefs, emotions and behavior of individuals influence the processes of understanding, communication and coping in the course of diagnosis and treatment. It has been proved [Salmon et al, 2009, pp. 450-456] that the patients want to communicate their needs that are linked with their desire for emotional support, though it happens infrequently; paradoxically, the clinical practice far too often continues the tradition of assuming that "doctor knows best" [Salmon, Hall 2004, pp. 53-56]. However, for centuries the problem of mental diseases was the dark side of medicine and the treatment of such persons was more than madness for the patients [Foucault 2006; Porter 2002; Shorter 2005; Shorter 1997].

Psychiatrists play a vital role in the patient's recovery: they help to achieve better therapeutic effects and are able to restore the patient's sense of security. In this way the treatment becomes more effective and the self-esteem increases. The change concerns not only quality but also the very meaning of life of persons with depressive disorders and with schizophrenia. Thus, patients are less inclined to consider the situation in which they find themselves (the disease and ways of treatment, including hospitalization) as a difficult situation.

\section{Material and methods}

The questionnaires were used to carry out the research. The study involved two groups of people: doctors and patients. 53 psychiatrists and 200 patients -78 with depressive disorders and 122 patients with schizophrenia were examined. The study was conducted in 2009, in all public mental hospitals in the region of Lodz. Each psychiatrist filled in two questionnaires: the first one - concerning patients with depression disorder, and the second one - relating to the patients suffering from schizophrenia. On the other hand, every patient filled in one questionnaire. Therefore, 106 questionnaires from the psychiatrists and 200 from the patients have become the base for the article. The talks with every examined person were additional sources of information.

The Bioethics Committee of the Medical University of Lodz, all the directors of hospitals and the heads of the appropriate hospital departments agreed to perform the tests in the hospitals. Every individual doctor and patient was also asked each time for consent to participate in the examination. Participation in the study was anonymous and voluntary. 
Almost the "mirror questionnaires" for the psychiatrists and for the patients were used in further stages of the research, in the aim to compare the collected material, or, in other words, to describe a problem from two sides. Most of the questions contained in the polls have been closed questions, but a few of them, being the most important for the subject matter, were allowed to be freely answered. Open questions are presented verbatim in order to avoid distortion and misinterpretation. In some cases, both doctors and patients could choose from a set of possible answers more than one answer that is why the results do not always add up to a hundred. The applied research method is standard in this type of research; however, for greater precision the extensive questionnaires were used. On key issues, respondents were asked about additional description (open questions).

Chi-square test was used for statistical analysis, and Pearson's chi-square test was used to verify the hypothesis significance. The study used a relative measure, that is, structure ratios (fractions). Basic statistical parameters: the median, the arithmetic mean and standard deviation, characterizing the properties of the tested population, and allowing for comparison with the other samples, were used for measurable (quantitative) characteristics.

Statistical analysis of the survey characteristics was performed on the basis of both parametric and non-parametric tests. Analysis of the empirical distribution of parameters was performed on the basis of the Shapiro-Wilk test. The application of parametric test was dependent on the assumption of the normal distribution and homogeneity of variance. The Leven test was applied to check the homogeneity of variance. In the analysis for qualitative characteristics two nonparametric tests were used: the Pearson chi-Square test for qualitative variables and Cramer's V test.

\section{The results of the study}

\section{Sociodemographic data}

$25 \%$ of women (13 doctors) and $75 \%$ of men ( 40 doctors) were reported in the group of the psychiatrists. In the group of patients the percentage of females and males was nearly the same: $51 \%$ : $49 \%$. The average age of patients with depression was 50 years and that of the patients with schizophrenia was 45 years. The median is 53 and 45 respectively. These differences are statistically significant $(\mathrm{p}<0.01)$. The youngest patient was a sixteen years old person suffering from depression; the youngest patient with schizophrenia was twenty years old. The oldest patients were at approximately the same age (73 and 72 years, respectively).

Most respondents (54\%) had a primary education or basic vocational education. $35 \%$ of surveyed patients had secondary or post-secondary education 
while $12 \%$ of respondents completed university studies. There were no significant differences in education levels between the two treatment groups of patients. The percentage of persons being still pupils or students was almost identical (10\%) among the two groups of patients. The same is true about the percentage of those who already completed their education. This may indicate either that the patients have not already belonged to the group of adolescents or that the disease could often make impossible the further education. In both groups of patients the majority (total $82 \%)$ was not economically active $(77 \%$ of people with depression and $85 \%$ of patients with schizophrenia). These results suggest two aspects of the same phenomenon: inactivity because of illness or immersing in one's own disease because of the lack of activity.

Living of a person with mental disease with his or her own family was by far the most common model which concerned $70 \%$ of respondents $(67 \%$ of people with depressive disorders and $72 \%$ of patients with schizophrenia). $32 \%$ of people with depressive disorders and $20 \%$ of patients with schizophrenia live alone; this is nearly a quarter of the total sample. Only $6 \%$ of surveyed patients live in nursing homes. This refers mainly to persons with schizophrenia (8\%) and only in one case relates to a patient with depressive disorders.

\section{Comparison of the results of doctors and patients' studies}

This section concerns the comparison of the positions represented by the two groups of respondents. Psychiatrists were asked for separate opinions pertaining to the patients with major depressive disorder and those with schizophrenia. As it turned out in most cases, the responses concerning both groups of patients usually do not differ significantly, so that doctors' answers can be considered as a whole relating to all patients. When the differences became statistically significant, it was included in the description. The same is true of the answers given by patients: the fundamental issue - their relationship with doctors - may be regarded as relatively homogeneous. We believe this fact is not entirely intuitive or trivial, more serious differences could perhaps be expected. And even though the answers provided by patients with depressive disorders and patients with schizophrenia usually did not substantially diverge, the differences between patients (considered as a whole) and the position of doctors were sometimes very significant.

In particular, opinions of doctors and patients relating their mutual contacts and information given by psychiatrists to their patients differed significantly from each other. Psychiatrists estimated that in 91\% of cases doctors were conducting their patients with major depressive disorder and $79 \%$ of patients with schizophrenia continuously (i.e. without interruption), while merely 38\% patients with depression and 20\% with schizophrenia confirmed that statement. Doctors claimed that during the first visit they had 
informed their patients about the specifics of the disease. The statement refers to $91 \%$ of people with depression and $83 \%$ of patients with schizophrenia. This position was confirmed by less than a half of the patients: $46 \%$ of patients with depression and $40 \%$ of those with schizophrenia. Psychiatrists also maintained that almost always they had informed the patients about the mode of action of individual drugs (after $92 \%$ of responses for each of the groups of patients), while only $64 \%$ patients with depression and $65 \%$ of patients with schizophrenia confirmed that opinion. Psychiatrists declared that they had informed patients (respectively $98 \%$ and $96 \%$ of responses) of the undesirable effects of drugs but only a half of patients (respectively $49 \%$ and $50 \%$ of patients) confirmed that. Convergent responses concerned two other issues. In the first case - "doctors answer all patients' questions" $-89 \%$ and $81 \%$ of psychiatrists' responses to the $83 \%$ and $77 \%$ of positive answers given by the patients were affirmative. The second case is related to the treatment. Both doctors and patients recognized that sometimes a decision is taken only by the psychiatrists and sometimes together with patients (respectively $58 \%$ and $53 \%$ of responses on the part of physicians and 55\% and $41 \%$ of the patients).

Doctors believed that patients presented them doubts and fears related to treatment $-92 \%$ of positive responses relating both to one and to the other of the diseases indicated by psychiatrists. This was confirmed by $71 \%$ of patients with major depressive disorder and $80 \%$ of patients with schizophrenia. Psychiatrists asserted (94\% of responses) that they sometimes changed the way of treatment at the request of the patient, which agrees with the answers given by the part of patients: $65 \%$ of them with major depressive disorder and $60 \%$ with schizophrenia. $87 \%$ of psychiatrists expressed the hope that their patients with depressive disorders had trusted them. As for the patients with schizophrenia, doctors showed more caution - at $77 \%$ positive responses. The same percentage, $87 \%$ of patients with depression and $86 \%$ of patients with schizophrenia (i.e. more that the doctors have expected) confirmed confidence in their doctor.

Psychiatrists estimated that at least two $(34 \%)$ or three $(28 \%)$ of ten patients with major depressive disorder who had started the treatment would interrupt it. That percentage may be even higher in patients with schizophrenia: three (28\%), four (34\%), and up to five patients $-24 \%$ (or even more) in ten who had begun the treatment could not endure to the end of therapy. Among the patients, $36 \%$ of persons with depression and $49 \%$ of patients with schizophrenia admitted they had stopped their treatment at least once in the past. Presumably, only a part of the patients responded truthfully, the rest did not have the courage to admit the break. This trend seems to confirm the statement of the doctors and patients relating to compliance. Doctors estimate that 
only $64 \%$ of patients with major depressive disorder and even less, i.e. $42 \%$ of patients with schizophrenia follow medical recommendations - as for the rest - they are not so sure. The patients' evaluation of the level of compliance was bigger than in the doctors' evaluation - respectively $92 \%$ and $91 \%$ of positive responses which is not astonishing. Doctors believed that their patients with depressive disorders considered their therapy as effective (96\%), while the persons with schizophrenia (in the doctors' opinion) determined the level of the effectiveness of the therapy as $72 \%$. Meanwhile, both groups of patients, assessed the treatment effect identically (after 77\% positive responses).

Differences were noticeable relating to the subject matter discussed in the psychiatric wards - the talks of psychiatrists and patients. Doctors claimed they had talked with their patients not only about their primary disease, but about all their life problems, what relates to absolutely all patients with major depressive disorder (100\% of responses on the part of psychiatrists), and $94 \%$ of patients with schizophrenia (as the doctors claim). Meanwhile, the above psychiatrists' opinion was confirmed by $72 \%$ of patients with major depressive disorder and merely $61 \%$ of patients with schizophrenia. This is one of the most glaring examples of disagreement between the doctors' and patients' evaluation. $75 \%$ of psychiatrists believe that the patient had been given enough time and attention, and $25 \%$ of doctors were not satisfied at this point with the facts. Meanwhile, $78 \%$ of people with major depressive disorder and to $83 \%$ with schizophrenia consider that their psychiatrists devoted them enough time and attention. Psychiatrists believe that almost all patients (100\% of responses relating to patients with depression and $98 \%$ of responses relating to patients with schizophrenia) were in contact with a doctor on time (during outpatient treatment). However, the psychiatrists' thesis is confirmed by just $71 \%$ of people with depression and $86 \%$ with schizophrenia.

One of the most serious problems in the treatment of both depression and schizophrenia is a stop or break in taking medication by patients without consulting their doctor. Resignation from taking medication usually has very negative impact on the effectiveness of a treatment. Therefore, two examined groups were asked about what is the reason for such decision. In the opinion of the psychiatrists the most common reason is the conviction of patients that they no longer need any medicine. There are $91 \%$ of doctors' indications relating to the patients with depression and $83 \%$ for the patients with schizophrenia. Also the patients have declared the same reason for discontinuation their pharmacotherapy. However, the difference between percentages is glaring: only half of them pointed out that reason $-52 \%$ of patients with major depressive disorder and $42 \%$ of patients with schizophrenia. The category "other reasons" (relating to the same problem: discontinuation the treatment) seems to be very interesting, because only a few doctors have pointed it out (respectively $6 \%$ and $9 \%$ 
of responses), but it was important for relatively many patients: $28 \%$ with depressive disorders and 25\% with schizophrenia. Patients have exchanged quite a few very serious reasons, such as loss of meaning in life and attempted suicide. The big problem among the patients is also the co-existence of alcoholism, which very often becomes the cause of the resignation of medication: patients, knowing that they must not combine drugs with alcohol, choose alcohol. The next major issue is the denial of illness or inability to accept it.

How important are these factors and the perceiving of these and other problems by the psychiatrists, can be proved by the fact that patients often decide to change the doctor just because they had no good contact with the previous one. This is confirmed by psychiatrists themselves, who declare that $91 \%$ of their patients with major depressive disorder and $89 \%$ of patients with schizophrenia have done it in the past. The patients, have confirmed opinion of the doctors although to a less extent (respectively 29\% and 30\%), but this can be explained by their fears that the doctor who was given up by them could have find it out.

Both doctors and patients notice a number of nuisances accompanying the process of treatment, although observations of doctors and patients seem to be considerably different. Psychiatrists believe, that the greatest inconvenience for the patients is the need for regular taking medicines, as evidenced by the $40 \%$ indications relating to patients with major depressive disorder and $62 \%$ relating to patients suffering from schizophrenia, while the patients located this factor in the third place adequately at a level of $9 \%$ (patients with depression) and $15 \%$ (patients with schizophrenia). Patients, recognized a hospitalization as the greatest nuisance, as indicated by $26 \%$ of persons with depression and $40 \%$ with schizophrenia. This factor is also cumbersome in the eyes of the doctors and was ranked in the second place, with indications of, respectively, $40 \%$ and $51 \%$. Limitations of the private life were recognized by patients as the second most nuisance factor (respectively $14 \%$ of people with depression and $26 \%$ of patients with schizophrenia). This drawback was also noted by the psychiatrists, who had described it at $38 \%$ relating to patients with major depressive disorder and $30 \%$ grappling with schizophrenia. In addition, doctors pointed to the need to comply with the rigors of treatment (respectively $32 \%$ and $47 \%$ ), which was reflected in patients only at the level of $3 \%$ and $11 \%$. Other factors do not seem to be so important for both physicians and patients.

Patients have presented two statements - "out of obviousness". First of all - as much as $30 \%$ of people with depression and $17 \%$ of patients with schizophrenia have found that nothing is burdensome for them in the course of treatment, and secondly - a very large proportion of patients, because $45 \%$ of major depressive disorder and 38\% suffering from schizophrenia have marked answer "other factors". Moreover, is not the first time, with a choice of a wide 
variety of categories, patients recognize that they do not fit in any of them, so that they had added their own arguments. Here we quote patients' explanations - what they understand by the term "other factors" without changes - in order to avoid distortion and misinterpretation. In their opinion, nuisance are: "Restriction of freedom (internment), the disease and impotence against it, difficult access to a doctor, too much distance between the place of residence and the place of treatment, side effects of medication, concerns about household affairs during the hospitalization, the inability of leisure time activities in the hospital, longing behind the house, the monotony of hospital life, lack of privacy and intimacy in the hospital, the lack of a pass to the house, the inability to contact the psychiatrist due to the permanent lack of time and interest on the part of the doctor, no effect of treatment, inability to accurate drug selection, unrealistic expectations for the effects of treatment, medication side effects - memory loss, reduction in private life, decreased libido, lack of companionship, life problems, inability to work, lack of medical discretion when patient is informed on his / her state of health, uncertainty about the effects of treatment, lack of exit for a walk, the continuing need to talk about themselves and their intimate affairs, co-morbidities, a significant limitation of personal freedom as a result of the treatment, keeping a secret from the family and the environment fact of staying in a mental hospital, the difficulty of getting to a doctor, hospital rhythm of life, necessity of compliance, medication side effects - drowsiness, family relationships, family breakdown as a result of the patient's illness, unemployment, stress, "gray days", the lack of social life, the stigma of mental illness in the environment - stigma, "excess" of drugs, too many patients the ward daily, no noticeable improvement in health, to mobilize against any effort, change doctors, lack of money for a decent life so, in addition to the purchase of medicines could be to go to the theater or to buy the book, the inability to implement prescriptions away from home, the failure of the hospital needed research, the disease - problems with memory, concentration, perception, hospitalization against the wishes of the patient, the disease hiding from the environment, large doses of medication, side effects of drugs - to reduce intellectual functioning, slow, fear, anxiety, irritability, lack of privacy and intimacy in hospital, dormitories (social phobia), incorrect diagnosis, incorrect medication". Patients' answer to the last question may seem surprising. Patients were asked whether, despite the numerous nuisances they accept their treatment. 93\% of them (patients with both depression and schizophrenia) answered affirmatively. This may prove strong determination to fight the disease and in the "private" fight for oneself.

Despite of the many differences in the perception of both the recovery and collaboration, doctors and patients are absolutely unanimous on one thing: a necessary condition of their cooperation is mutual trust and respect. Patients 
are willing to sacrifice a lot, provided, however, that the doctors saw in them people, not the statistical number of diseases. Also, doctors are willing to make concessions, but on the condition of sincerity on the part of patients. Thus, the mutual co-operation seems to be possible.

Each group is aware of the many barriers of their communication, but both sides are willing to understand each other and cooperate.

\section{Conclusions}

1. Both doctors and patients are in agreement as to the basic element of good cooperation that is mutual trust.

2. However, doctors and patients understand the concept of mutual cooperation quite differently. This difference is due to the other experiences: psychiatrists use their knowledge and years of practice, patients - experiences from the period of the disease and the treatment; additionally their perception of reality could be distorted as the result of the disease.

3. Psychiatrists claim that they provide comprehensive information on both disorders, which their patients suffer from, as well as methods of treatment, and adverse effects of drugs; however, no more than a half of the patients confirm that. Both the persons with depression and schizophrenia maintain that they follow medical recommendations, but in the opinion of doctors this is true only about a part of the patients.

4. The level of compliance in depressive disorders was evaluated by psychiatrists as $64 \%$ and among the patients with schizophrenia as $42 \%$ (when 92\% depressive patients and 91\% persons with schizophrenia declare compliance by themselves). Differences between both groups of patients are substantial statistically $(\mathrm{p}<0.05)$, indicating that more persons with major depressive disorder follow medical recommendations than patients with schizophrenia, at least in the doctors' opinion.

5. According to the doctors, the most common reason for the desisting from taking medications by patients (both with major depressive disorder and schizophrenia - adequately: $91 \%$ and $83 \%$ ), is their belief that drugs are no longer needed. Patients confirmed the opinion of the psychiatrists (claiming they have no benefits of pharmacotherapy), but only 52\% of persons with major depressive disorder and $42 \%$ with schizophrenia drew attention to this.

6. Both psychiatrists and patients themselves - notice a number of nuisances accompanying the process of treatment. However - observations of doctors and patients do not fully coincide. 
7. Doctors as well as their patients indicate numerous barriers of interpersonal communication meanwhile declaring attempts of understanding and mutual cooperation.

8. Psychiatrists and patients agree that the quality of the relationship, proper contact and cooperation affect mental disposition and patient motivation for the process of recovery from the illness, the treatment and the proper social functioning of both groups of patients.

\section{Inference:}

Psychiatrists should explain to the patients what is the overarching goal of therapy. Doctors and patients should jointly discuss medical procedures and psychological problems associated with the treatment. The result of cooperation should be the conclusion of a contract between a psychiatrist and patient. Each party shall abide by its commitments.

\section{Bibliography}

Beach M.C., Roter D.L., Wang N.Y. et al. (2006), Are physicians' attitudes of respect accurately perceived by patients and associated with more positive communication behaviors?, 'Patient Educ Couns', 62 (3), pp. 347-354.

Foucault M. (2006), History of Madness, Routledge, London, New York.

Hill G. (2011), Seven steps to successful organizational collaboration, Optima Partners, Edinburgh.

Morgan J. (2012), The Collaborative Organization: A Strategic Guide to Solving Your Internal Business Challenges Using Emerging Social and Collaborative Tools, McGraw-Hill, New York.

Porter R. (2002), Madness. The Brief History, Oxford University Press, Oxford, New York.

Pulia M. (2011), Simple Tips to Improve Patient Satisfaction, 'American Academy of Emergency Medicine', 18 (1), pp. 18-19.

Roter D.L. (1977), Patient participation in the patient-provider interaction: the effects of patient question asking on the quality of interaction, satisfaction and compliance, 'Health Educ Monogr', 5 (4), pp. 281-315.

Roter D.L., Frankel R.M., Hall J.A. et al. (2006), The expression of emotion through nonverbal behavior in medical visits. Mechanisms and outcomes, 'J Gen Med', 21 Suppl 1, pp. S28-34.

Roter D.L., Hall J.A., Katz N.R. (1987), Relations between physicians' behaviors and analogue patients' satisfaction. Recall, and impressions, 'Med Care', 25 (5), pp. 437-451. 
Salmon P. (2006), Explaining unexplained symptoms: the role of beliefs in clinical management [in:] P. Halligan, M.Aylward (ed.), The power of belief, Oxford University Press, Oxford, pp. 137-159.

Salmon P. (2000), The Psychology of Medicine and Surgery: A Guide for Psychologists, Counsellors, Nurses and Doctors, Wiley Series in Clinical Psychology, Chichester.

Salmon P., Hall G.M. (2004), Patient empowerment or the emperor's new clothes, JRSM, 97 (2), pp. 53-56.

Salmon P., Ring A., Humpheris G.M. et al. (2009), Primary Care Consultations About Medically Unexplained Symptoms: How Do Patients Indicate What They Want?, JGIM, 4, pp. 450-456.

Shorter E. (2005), A Historical Dictionary of Psychiatry, Oxford University Press, Oxford, New York.

Shorter E. (1997), A History of Psychiatry: From the Era of Asylum to the Age of Prozac, John Wiley \& Sons Inc., New York.

Sofaer S., Firminger K. (2005), Patient perceptions of the quality of health services, "Annual Review of Public Health", 26, pp. 513-559.

World Health Organization (2004), Promoting Mental Health. http://www.who. int/mental_health/evidence/en/promoting_mhh.pdf [17.09.2013]. 